\title{
PREPARATION AND IN VITRO CHARACTERIZATION OF ACETAMINOPHEN BY SOLID SOLUTION TECHNIQUE
}

\author{
*Shashank Soni ${ }^{1}$, Deepa Dhiman ${ }^{1}$ \\ Department of Pharmaceutics, Smt. Tarawati Institute of Bio medical and allied sciences, Roorkee, India \\ *Correspondence author's E-mail: shashank_soni64@yahoo.com, Contact no: +919410572306
}

Received 21 July 2012; Review Completed 10 Aug 2012; Accepted 26 Aug 2012, Available online 15 Sep 2012

\begin{abstract}
Paracetamol (PCM) is poor water soluble drug which comes under Biopharmaceutical classification system (BCS) class III, having ( $\log \mathrm{p}=0.5$ ) and shows dissolution rate limited absorption. The solid dispersion of this using PEG 4000 as a carrier in different ratio prepared by using trituration method and solidification technique followed by a formation of a solid solution phase. The prepared dispersions characterized for a solubility study, drug content analysis, in-vitro release profile and stability study. The drug content was found to be high in formulation F4 due to the high carrier ratio which causes the creation of a highly hydrophilic environment. The stability study of formulated dispersions also explains the PEG 4000 is a better choice for this study and does not changes its physical as well as chemical property on being storage. The prepared dispersion by this technique improved the dissolution rate as well as solubility of the prepared dispersion when compared with the raw drug (RD) and physical mixture (PM).
\end{abstract}

Key words: Paracetamol, BCS class III, PEG 4000, Solid solution

\section{INTRODUCTION}

The term solid dispersion refers to a group of solid products consisting of at least two different components, generally a hydrophilic matrix and a hydrophobic drug. The matrix can be either crystalline or amorphous. The drug can be dispersed molecularly, in amorphous particles (clusters) or in crystalline particles ${ }^{1}$.

Enhancement of bioavailability of poorly soluble drug has become draustic need and challenging aspects for the drug development phase ${ }^{2}$. For the better absorption of the drug it is quite necessary that drug must be soluble and permeable which decides the bioavailability of the drug. So the formulation of poorly soluble drug for the drug delivery has becoming challenging to the research and development sectors and work is going through on this.

So in this case the major approach is the formulation approach which is quite simple and increases the solubility of the poorly soluble drug by the solid dispersion technique. This method, which was later, termed solid dispersion which involved the formation of eutectic mixture of drugs with water-soluble carriers by the melting of their physical mixtures.

On the basis generation solid dispersion broadly classified into ${ }^{3}$ -

1. First generation solid dispersions- include crystalline carriers such as urea ${ }^{4,5}$ and sugars ${ }^{6}$.

2. Second generation solid dispersions- includes polymeric carriers which are purely synthetic like Povidone $(\mathrm{PVP})^{[7]}$, Polyethylene glycol (PEG) ${ }^{\mathbf{8}}$, polymethylacrylates 9 , natural like cellulose derivatives, such as hydroxypropylmethylcellulose $(\text { HPMC })^{10}$ ethyl cellulose ${ }^{11}$ or hydroxypropylcellulose or starch derivates, like cyclodextrins ${ }^{[12]}$.

3. Third generation solid dispersions- includes blends of surfactant and polymers like inulin, inutec SP1, compritol $888 \mathrm{ATO}^{\mathbf{1 3}}$, mixture of PEG and polysorbate 80, HPMC was also associated with poloxamer and polyoxyethylene hydrogenated castor oil to prepare an amorphous

felodipine solid dispersion.

\section{MATERIALS}

Paracetamol (PCM) was gifted by Simpex Laboratories Kotdwar, India. PEG 4000, buffers was purchased from $\mathrm{CDH}$, and all other chemicals used were of analytical grade.

\section{METHODS}

\section{Preparation of physical mixture}

The physical mixture of PCM and PEG 4000 was accurately taken in a mortar and triturated smoothly with the help of pestle so that mixing makes uniformly, drug and carrier mix with each other and distributed equally in both the phases. The resulting mixture formed was passed through the sieve no.80. The mixture was placed in a desiccator to carry the further characterization of prepared mixture.

\section{Preparation of solid dispersion of PCM containing PEG 4000 as a carrier}

Firstly the carrier PEG 4000 was melted in a china dish over a water bath at temperature $55{ }^{\circ} \mathrm{C}$, then the accurately amount of a PCM was incorporated in a molten carrier and with the help of glass rod, solid solution formed was properly mixed so that uniform mixing takes place. After that transfer the china dish over an ice bath and solidify the molten mixture, put this system into a desiccator fitted with vaccum remove the moisture from it, passed with the sieve and when use transfer in a capsule and evaluation was performed. 
Table 1: Formulation composition for PCM solid dispersion containing PEG 4000 as a carrier

\begin{tabular}{|c|c|c|}
\hline Formulation Code & Carrier (PEG 4000) & PCM \\
\hline RD & - & $500 \mathrm{mg}$ \\
\hline F1* & $100 \mathrm{mg}$ & $500 \mathrm{mg}$ \\
\hline F1 & $100 \mathrm{mg}$ & $500 \mathrm{mg}$ \\
\hline F2 & $200 \mathrm{mg}$ & $500 \mathrm{mg}$ \\
\hline F3 & $300 \mathrm{mg}$ & $500 \mathrm{mg}$ \\
\hline F4 & $400 \mathrm{mg}$ & $500 \mathrm{mg}$ \\
\hline
\end{tabular}

\section{Characterization of PCM solid dispersions}

Saturation solubility studies of prepared solid dispersions

The excess amount of solid dispersions (physical mixtures and prepared dispersion) was added to the $10 \mathrm{ml}$ volumetric flask which contains distilled water and subjected to kept for maintaining $37{ }^{0} \mathrm{C}$ for 26 hours. Then the flask were removed and suitable amount of aliquots was taken and filtered by using whattmann filter paper and diluted by using distilled water and it is compare with the pure drug for solubility profile comparison.

\section{Appearance of Paracetamol solid dispersion}

By the help of projection microscope at magnification $10 \mathrm{x}$ and $40 \mathrm{x}$ the prepared PCM dispersion were analyzed to study the phase transformation between the states.

\section{Drug content analysis}

Preparation equivalent to $100 \mathrm{mg}$ was accurately weighed and transferred to $100 \mathrm{ml}$ volumetric flask and mixed in $100 \mathrm{ml}$ phosphate buffer having $\mathrm{pH}$ 5.8., suitable aliquots were withdrawn and diluted to appropriate dilution and drug content was established using calibration curve of PCM at $\lambda_{\max } 243 \mathrm{~nm}$. The drug content was estimated.

\section{In - vitro release profile characteristics}

In-vitro release of PCM from the solid solution were evaluated with a USP XXXI dissolution apparatus type II (paddle type, Electrolab, Mumbai, India) at $50 \mathrm{rpm}$ in $900 \mathrm{ml}$ phosphate buffer $(\mathrm{pH} 5.8)$ at $37 \pm 0.5^{\circ} \mathrm{C}$. At predetermined intervals, a $1 \mathrm{ml}$ aliquot was withdrawn and replenished with an equal volume of fresh dissolution medium. The withdrawn samples were analyzed spectrophotometrically at $243 \mathrm{~nm}$ PCM solid dispersion formulations.

\section{Kinetics of drug release}

The results of in vitro release profiles obtained for all the formulations were fitted into four models of data treatment as follows:

1. Cumulative percent drug released versus time (zeroorder kinetic model).

2. Log cumulative percent drug remaining versus time (first-order kinetic model).

3. Cumulative percent drug released versus square root of time (Higuchi's model).

4. Log cumulative percent drug released versus log time (Korsemeyer-Peppas equation).

\section{Stability studies}

The stability studies of selected formulation was carried out at the room temperature in a hermitically sealed ampoules for 6 months to observe any change in physical characteristics, drug content and whether any drug release profile alters or not while storage.

\section{RESULTS AND DISCUSSION}

Callibration curve of Paracetamol in $5.8 \mathrm{pH}$ phosphate buffer

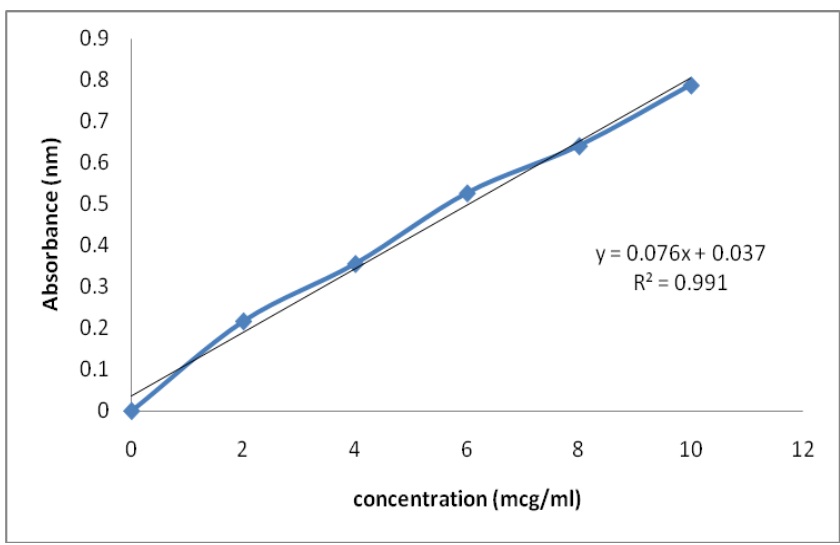

Figure 1: Callibration curve of Paracetamol in $5.8 \mathrm{pH}$ phosphate buffer at $243 \mathrm{~nm}$

Saturation solubility studies of prepared solid dispersions

The solubility profile of solid dispersion, physical mixture and pure drug was found to be and depicted in the following table 2 .

Table 2 Solubility study table in different ratio of carrier

\begin{tabular}{|c|c|c|c|}
\hline Formulation Code & Method & Carrier & $\begin{array}{c}\text { Solubility in Water mg/ml } \\
(\mathbf{(} \mathbf{S} . \mathbf{D}, \mathbf{n}=\mathbf{3})\end{array}$ \\
\hline RD & - & PEG 4000 & $0.079 \pm 0.002$ \\
\hline F1 & Trituration & PEG 4000 & $0.102 \pm 0.016$ \\
\hline F1 & Melting & PEG 4000 & $0.204 \pm 0.015$ \\
\hline F2 & Melting & PEG 4000 & $0.219 \pm 0.023$ \\
\hline F4 & Melting & PEG 4000 & $0.245 \pm 0.014$ \\
\hline
\end{tabular}

Paracetamol is purely water insoluble drug and its solubility at room temperature in pure distilled water was estimated $0.079 \mathrm{mg} / \mathrm{ml}$. But the dispersion of PCM with PEG 4000 proves a better solubility profile and increases the solubility of PCM with as the carrier proportion (c) 2011, JDDT. All Rights Reserved increases. In formulation $\mathrm{F} 1 *$ there is marked increase of solubility of PCM with physical mixture of PEG 4000 by trituration method, because there is less intimate between the drug and carrier so this not so affect the solubility of $\mathrm{PCM}$ in physical mixture $(\mathrm{F} 1 *)$.

ISSN: 2250-1177 
In the another formulations (F1 to F4), the dispersion prepared by the melting solidification technique so in this there is may be inherent transitions takes place between the drug and carrier in manner of hydration, increase in wettability phenomenon and possible complex formation with the carriers which leads to increase in the solubility ranging from $0.204 \mathrm{mg} / \mathrm{ml}$ to $0.253 \mathrm{mg} / \mathrm{ml}$.
Pure form of PCM having the crystalline nature where as the physical mixture of PEG:PCM having the off sticky appearance and the prepared solid dispersion of formulation shows the solid sticky lumps at temperature 37 ${ }^{\circ} \mathrm{C}$ but at above this temperature it starts melting and physical property on the basis of integrity of dispersions it starts changing as the temperature and time progress.

\section{Appearance of Paracetamol solid dispersion}

Table 3: Appearance of solid dispersion formulation

\begin{tabular}{|c|c|c|}
\hline Formulation Code & Method & Nature of formulation \\
\hline RD & - & White crystalline powder \\
\hline F1 & Trituration & Off sticky texture \\
\hline F1 & Melting & Solid sticky lumps \\
\hline F2 & Melting & Solid sticky lumps \\
\hline F3 & Melting & Solid sticky lumps \\
\hline F4 & Melting & Solid sticky lumps \\
\hline
\end{tabular}

\section{Drug content analysis}

Preparation equivalent to $100 \mathrm{mg}$ was accurately weighed and transferred to $100 \mathrm{ml}$ volumetric flask and mixed in $100 \mathrm{ml}$ phosphate buffer having $\mathrm{pH}$ 5.8., suitable aliquots were withdrawn and diluted to appropriate dilution and drug content was established using calibration curve of PCM at $\lambda_{\max } 243 \mathrm{~nm}$. The drug content was found to be between $93.78 \%$ to $99.04 \%$ as compared with respect to the standard one. This explains that the drug is properly dispersed in the carrier phase which shows the higher significance value.

\section{In vitro release profile study}

The formulation of solid dispersions with carrier like PEG 4000 was formulated and proves a better release profile at pH 5.8 phosphate buffer. However it was also experimented and observed that the prepared solid dispersions with these carriers shows a better release characteristics as compared with the raw drug (RD) and physical mixture $(\mathrm{F} 1 *)$. Formulation $\mathrm{F} 4$ reveals the faster dissolution rate as compared to the other formulations because in this the ratio of carrier is high and this leads to the faster release of this formulation. From the release graph which was plotted against the time also shows there is slowly release of the drug from this carrier because at the earlier of the few minutes there is not enough time to get between the carrier and media to increase the wettability of the carriers and as the time progresses the wetability increases and this finally leads to the better release of the drug.

These observations however indicates that the better and enhanced dissolution of the dispersions is due to the increased wettability of the drug and carrier, particle size reduction in the case of during preparation and phase transformation between these two.

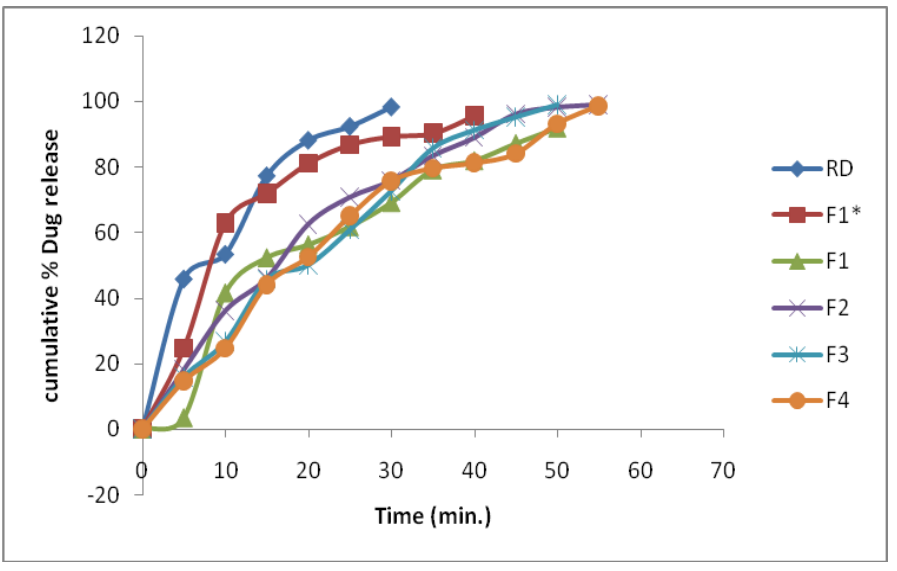

Figure 2: Release profiles of various formulations at
pH 5.8 phosphate buffer

Release kinetics of the solid dispersions

The in vitro release kinetics of various formulations was analyzed by fitting the various models as mentioned in the above table. While establishing the release kinetics with various formulations it was found that the formulation $\mathrm{RD}$, $\mathrm{F} 1 *, \mathrm{~F} 1, \mathrm{~F} 2$ and $\mathrm{F} 4$ fallows a zero order kinetics because in this calculated $r^{2}$ value were higher and it explains that the release rate is independent of the concentration of the drug. While establishing connection to the release kinetics in F3 it was observed that the $r^{2}$ value in Higuchi role model was higher and it reveals that the solid drug is dispersed in a soluble matrix and the rate of drug release is related to the rate of drug diffusion. The $\mathrm{n}$ values from drug release experiment ranged from $0.58-0.78$, indicated anomalous non-Fickian transport, which suggest that mechanism and kinetics of drug release were dependent on the solubility of solid dispersion formulation in dissolution medium.

Table 4: Release kinetics of the solid dispersion

\begin{tabular}{|c|l|l|l|l|c|}
\hline \multirow{2}{*}{ Formulation Code } & \multicolumn{3}{|c|}{$\mathbf{r}^{2}$ Value } & \multicolumn{1}{c|}{ Korsmeyer-Peppa's value } \\
\cline { 2 - 6 } & \multicolumn{1}{|c|}{ Zero order } & First order & \multicolumn{1}{|c|}{ Higuchi } & 0.9227 & 0.76 \\
\hline RD & 0.9920 & 0.7302 & 0.9106 & 0.5431 & 0.60 \\
\hline F1* & 0.9991 & 0.6758 & 0.8659 & 0.9777 & 0.60 \\
\hline F1 & 0.9791 & 0.6797 & 0.9231 & 0.9897 & 0.77 \\
\hline F2 & 0.9957 & 0.7915 & 0.9116 & 0.9974 & 0.58 \\
\hline F4 & 0.9382 & 0.5548 & 0.9839 & 0.9563 & 0.78 \\
\hline
\end{tabular}




\section{Stability studies of the solid dispersions}

In the present contrast of study we have done some extent of stability study as per the established guidelines. During this the prepared dispersions were sealed in hermitically sealed containers (ampoules) of different formulations. After storage at room conditions for six months the prepared solid dispersions were characterized for physical appearance, solubility studies, drug content analysis and dissolution profile studies.

It was found that there is no any much more changes occur to the solid dispersions formulations with using PEG 4000 as a carrier. However there is much more study needed during the stability studies of dispersion by following the ICH guidelines.

\section{CONCLUSION}

In this present study we have prepared the solid dispersion of Paracetamol using PEG 4000 as a carrier. Due to

\section{REFERENCES}

1. Chiou WL and Riegelman S, Preparation and dissolution characteristics of several fast-release solid dispersions of Griseofulvin, J. Pharm. Sci.,1969, 58(12), 1505-1510

2. Akiladevi D, Shanmugapandiyan P, Jebasingh D, Basak D, Preparation and evaluation of Paracetamol solid dispersion, International journal of pharmacy and pharmaceutical sciences, 2011, 3(1), 188-191

3. Vasconcelos T, Sarmento B and Costa P, Solid dispersion as strategy to improve oral bioavailability of poor water soluble drugs, Drug discovery today, 2007, 12, 1068-1075

4. Sekiguchi K and Obi N, Studies on Absorption of Eutectic Mixture. Absorption of Fused Conglomerates of Chloramphenicol and Urea in Rabbits, Chem. Pharm. Bull (Tokyo), 1964, 12, 134-144

5. Sekiguchi K, and Obi N, Studies on absorption of eutectic mixtures. A comparison of the behavior of eutectic mixtures of sulphathiazole and that of ordinary sulphathiazole in man, Chem. Pharm. Bull (Tokyo), 1961, 9, 866-872

6. Kanig J.L, Properties of Fused Mannitol in Compressed Tablets, J. Pharm. Sci., 1964, 58, 188-192

7. Karavas E, Application of PVP/HPMC miscible blends with enhanced mucoadhesive properties for adjusting drug hydrophilic nature of this carrier molecule this supports the creation of hydrophilic environment to the dispersion solid and increases the wettability of the Paracetamol which leads to increase in the dissolution rate. In this study we observe that by increasing the concentration of carrier the dissolution and solubility profile also increases. This carrier also proves the better in terms of stability. We propose that the prepared solid dispersion using PEG 4000 was able to improve the solubility and dissolution profile of poorly water soluble drug without changing in its integrity.

\section{ACKNOWLEDGEMENT}

Authors are thankful to Dr. Pankaj Sharma, Director of Smt. Tarawati institute of Bio medical and allied sciences, Roorkee, India for providing institutional funds to carry out the research work. Authors are also thankful to Simpex Laboratories Kotdwar, India, for providing a gift sample of Paracetamol in time. release in predictable pulsatile chronotherapeutics, Eur. J. Pharm. Biopharm, 2006, 64, 115-126

8. Urbanetz N.A, Stabilization of solid dispersions of nimodipine and polyethylene glycol 2000. Eur. J. Pharm. Sci., 2006, 28, 67-76

9. Huang J, Nifedipine solid dispersion in microparticles of ammonio methacrylate copolymer and ethylcellulose binary blend for controlled drug delivery: Effect of drug loading on release kinetics, Int. J. Pharm, 2006, 319, 44-54

10. Konno $\mathrm{H}$ and Taylor L.S, Influence of different polymers on the crystallization tendency of molecularly dispersed amorphous felodipine, J. Pharm. Sci., 2006, 95, 2692-2705

11. Desai J, Characterization of polymeric dispersions of dimenhydrinate in ethyl cellulose for controlled release, Int $\mathbf{J}$ Pharm, 2006, 308, 115-123

12. Garcia $X$ and Zubiri I, Thermal stability of solid dispersions of naphthalene derivatives with [beta]-cyclodextrin and [beta]-cyclodextrin polymers. Thermochim. Acta, 2006, $444,57-64$

13. Li F, In vitro controlled release of sodium ferulate from Compritol 888 ATO-based matrix tablets, Int J Pharm, 2006, $324,152-15$. 